\title{
Is a global coupled-channel dispersive optical model potential for actinides feasible?
}

\author{
R. Capote, ${ }^{1, *}$ E. Sh. Soukhovitskii, ${ }^{2}$ J. M. Quesada, ${ }^{3}$ and S. Chiba ${ }^{4}$ \\ ${ }^{1}$ Nuclear Data Section, Division of Physical and Chemical Sciences, Department of Nuclear Sciences and Applications, \\ International Atomic Energy Agency, Vienna A-1400, Austria \\ ${ }^{2}$ Joint Institute for Energy and Nuclear Research, 220109, Minsk-Sosny, Belarus \\ ${ }^{3}$ Departamento de Física Atómica, Molecular y Nuclear, Universidad de Sevilla, Ap. 1065, E-41080 Sevilla, Spain \\ ${ }^{4}$ Advanced Science Research Center, Japan Atomic Energy Agency, 2-4 Shirakata Shirane, Tokai, Naka, Ibaraki 319-1195, Japan
}

(Received 2 September 2005; published 15 December 2005)

\begin{abstract}
An isospin-dependent coupled-channel optical model potential containing a dispersive term with a nonlocal contribution is used to simultaneously fit the available experimental database (including strength functions and scattering radius) for neutron and proton scattering on strongly deformed ${ }^{238} \mathrm{U}$ and ${ }^{232} \mathrm{Th}$ nuclei. The energy range 0.001-200 MeV is covered. A dispersive coupled-channel optical model (DCCOM) potential with parameters that show a smooth energy dependence and energy-independent geometry are determined from fits to the entire data set. Calculations using the obtained DCCOM potential reproduce measured total cross-section differences between ${ }^{232} \mathrm{Th}$ and ${ }^{238} \mathrm{U}$ within experimental uncertainty. The isovector terms and the observed very weak dependence of the geometrical parameters on mass number $A$ allow us to extend the derived potential parameters to neighboring actinide nuclei with great confidence.
\end{abstract}

DOI: 10.1103/PhysRevC.72.064610

PACS number(s): 11.55.Fv, 24.10.Ht

\section{INTRODUCTION}

It is generally accepted that differences of neutron total cross sections among neighboring nuclei provide an unusually stringent test of optical models [1-4]. At the same time it has been shown that the standard optical model treatment fails to reproduce the observed differences of total cross sections both for tungsten isotopes [4] and Th-U nuclei [5]. Based on these findings it was stated that the prediction of the global optical model potential for actinides with $A \geqslant 232$ still needs extensive experimental and theoretical investigations [5]. We show that an isospin-dependent coupled-channel (CC) optical model potential (OMP) containing a dispersive term including nonlocal contribution is a very good candidate to be used as a global actinide optical model potential.

Recent high-precision measurements of the neutron total cross sections for ${ }^{232} \mathrm{Th}$ and ${ }^{238} \mathrm{U}$ nuclei at energies from 5 up to $560 \mathrm{MeV}$ were published by a LivermoreLos Alamos-Ohio University collaboration [6]. The crosssection difference data are presented as the ratio of the measured difference to the average of the individual cross sections: i.e., as $R^{238} \mathrm{U}-232 \mathrm{Th}=\left[\sigma\left({ }^{238} \mathrm{U}\right)-\sigma\left({ }^{232} \mathrm{Th}\right)\right] /\left\{\left[\sigma\left({ }^{238} \mathrm{U}\right)+\right.\right.$ $\left.\left.\sigma\left({ }^{232} \mathrm{Th}\right)\right] / 2\right\}$. We use these data as a test of a newly derived dispersive coupled-channel optical model (DCCOM), based on all available nucleon-scattering information for both nuclei.

It has been shown that consideration of dispersion effects allows us to describe both bound and scattering states by the same nuclear mean field [7-12]. Pioneering works on dispersive optical model (DOM) analysis for nucleon scattering were done by Lipperheide [13,14], Passatore [15], and Lipperheide and Schmidt [16]. Recently a global dispersive spherical potential for neutron-induced reactions was derived by Morillon and Romain [17]. However, very few studies have been devoted to dispersive OPMs for strongly deformed

*Electronic address: R.CapoteNoy@iaea.org nuclei, in which CC formalism should include dynamical potential corrections arising from dispersion effects [18-22]. Most of the CC potentials suggested for actinides are based on the potentials determined by Lagrange [23]. A new global parametrization using nondispersive CC OMPs for actinide nuclei valid from $1 \mathrm{keV}$ to $200 \mathrm{MeV}$ was recently proposed by Soukhovitskiĩ and co-workers [24] (in the following text we refer to this work as NDOMP04). CC OMPs describing nucleon scattering on ${ }^{238} \mathrm{U}$ nucleus up to $150-200 \mathrm{MeV}$ became recently available $[25,26]$. During the past year, several new OMPs for thorium are being discussed within the framework of the on going International Atomic Energy Agency Coordinated Research Program [27], including those derived by Maslov [5] and Ignatyuk and co-workers [28].

We construct a deformed complex mean field felt by neutrons and protons in ${ }^{232} \mathrm{Th}$ and ${ }^{238} \mathrm{U}$ theoretically valid from the Fermi energy up to 200-MeV energy. The methodology was outlined in our previous contribution devoted to the study of the ${ }^{232} \mathrm{Th}$ nucleus [22]. The real and imaginary parts of the mean field are connected by a dispersion relation, and, moreover, the mean field is required for closely reproducing the experimental value of the Fermi energy $E_{F}$ both for neutrons and protons. The Lane model $[29,30]$, which assumes isospin symmetry in nuclei, is employed so our nucleon-nucleus OMP can be decomposed into isoscalar and isovector parts.

\section{COUPLED-CHANNEL DISPERSIVE OPTICAL MODEL FORMALISM}

Actinides are expected to be well-deformed rigid rotors, in which low-lying collective levels are strongly excited in nucleon inelastic scattering. We performed the customary CC calculations by coupling the first five states of the ground-state $K^{\pi}=0^{+}$rotational band, $J^{\pi}=0^{+}, 2^{+}, 4^{+}, 6^{+}$, and $8^{+}$. Our analysis spans an energy range from 0.001 up to $200 \mathrm{MeV}$. Both 
direct and statistical processes contribute to nucleon-nucleus elastic scattering at these energies. However, according to our estimation, the statistical processes are not important above $3 \mathrm{MeV}$ so we neglect them in the OMP derivation. The direct processes, increasingly dominant at higher energies, can be described by the optical model.

The deformed nuclear optical potential arises from deformed instant nuclear shapes,

$$
\begin{gathered}
R_{i}\left(\theta^{\prime}, \varphi^{\prime}\right)=R_{i}^{0}\left[1+\sum_{\lambda=2,4,6,8} \beta_{\lambda 0} Y_{\lambda 0}\left(\theta^{\prime}, \varphi^{\prime}\right)\right] \\
i=\mathrm{HF}, v, s, \text { so }, c
\end{gathered}
$$

where $Y_{\lambda 0}$ means spherical harmonics, $\theta^{\prime}$ and $\varphi^{\prime}$ are angular coordinates in the body-fixed system, $v, s$, so, and $c$ are the volume, surface, spin-orbit, and Coulomb terms, respectively and $R_{i}^{0}=r_{i} A^{1 / 3}$, where $A$ is the target mass number. The HF index corresponds to the smooth real volume potential (usually called the Hartree-Fock term).

The OMP is taken to be a standard Woods-Saxon form, but with account of the deformed nuclear shapes. A more general formulation than the one used in our previous work [22] is described below. The OMP potential may be written as

$$
\begin{aligned}
V & {\left[r, R\left(\theta^{\prime}, \varphi^{\prime}\right), E\right] } \\
& =-\left[V_{\mathrm{HF}}(E)+\Delta V_{\mathrm{HF}}^{\mathrm{Coul}}(E)\right] f_{\mathrm{ws}}\left[r, R_{\mathrm{HF}}\left(\theta^{\prime}, \varphi^{\prime}\right)\right] \\
& -\left[\Delta V_{v}(E)+\Delta V_{v}^{\mathrm{Coul}}(E)+i W_{v}(E)\right] f_{\mathrm{ws}}\left[r, R_{v}\left(\theta^{\prime}, \varphi^{\prime}\right)\right] \\
& -\left[\Delta V_{s}(E)+\Delta V_{s}^{\mathrm{Coul}}(E)+i W_{s}(E)\right] g_{\mathrm{ws}}\left[r, R_{s}\left(\theta^{\prime}, \varphi^{\prime}\right)\right] \\
& +\left(\frac{\hbar}{m_{\pi} c}\right)^{2}\left[V_{\mathrm{so}}(E)+\Delta V_{\mathrm{so}}(E)+i W_{\mathrm{so}}(E)\right] \\
& \times \frac{1}{r} \frac{d}{d r} f_{\mathrm{ws}}\left[r, R_{\mathrm{so}}\left(\theta^{\prime}, \varphi^{\prime}\right)\right](\hat{\sigma} \cdot \hat{L})+V_{\mathrm{Coul}}\left[r, R_{c}\left(\theta^{\prime}, \varphi^{\prime}\right)\right]
\end{aligned}
$$

where the first term is the real smooth volume potential $V_{\mathrm{HF}}(E)$ and its corresponding Coulomb correction $\Delta V_{\mathrm{HF}}^{\mathrm{Coul}}(E)=$ $-C_{\mathrm{Coul}} \frac{Z Z^{\prime} e^{2}}{A^{1 / 3}} \frac{d}{d E}\left[V_{\mathrm{HF}}(E)\right]$. Similar Coulomb correction terms $\Delta V_{v}^{\text {Coul }}(E)$ and $\Delta V_{s}^{\text {Coul }}(E)$ are also calculated for volume $\Delta V_{v}(E)$ and surface $\Delta V_{s}(E)$ dispersive contributions to the real potential. Successive complex-valued terms are the volume, surface, and spin-orbit potentials, all containing the corresponding dispersive contributions $\Delta V_{v}(E), \Delta V_{s}(E)$, and $\Delta V_{\text {so }}(E)$. The geometrical form factors are given as

$$
\begin{aligned}
f_{\mathrm{ws}}\left[r, R_{i}\left(\theta^{\prime}, \varphi^{\prime}\right)\right] & =\left\{1+\exp \left[r-R_{i}\left(\theta^{\prime}, \varphi^{\prime}\right)\right] / a_{i}\right\}^{-1}, \\
i & =\mathrm{HF}, v, \text { so, } \\
g_{\mathrm{wS}}\left[r, R_{s}\left(\theta^{\prime}, \varphi^{\prime}\right)\right] & =-4 a_{s} \frac{d}{d r} f\left[r, R_{s}\left(\theta^{\prime}, \varphi^{\prime}\right)\right]
\end{aligned}
$$

where deformed radii $R_{i}\left(\theta^{\prime}, \varphi^{\prime}\right)$ are described in Eq. (1). The Coulomb potential $V_{\text {Coul }}\left[r, R_{c}\left(\theta^{\prime}, \varphi^{\prime}\right)\right]$ was calculated with a multipole expansion of charged ellipsoid with a uniform charge density within the Coulomb radius $R_{c}$ and zero outside, as suggested by Bassel et al. [31]. We calculated the spherical term of the Coulomb potential, taking into account the diffuseness of the charge-density distribution of the form $f_{c}=\left[1+\exp \left(r-R_{c}^{0}\right) / a_{c}\right]^{-1}[32]$.
In our formulation of the OMP in Eq. (2) the geometrical parameters of the Hartree-Fock (HF) potential $r_{\mathrm{HF}}$ and $a_{\mathrm{HF}}$ are in general different from geometrical parameters $r_{v}, a_{v}, r_{s}$, and $a_{s}$ of the volume and surface absorptive potentials; however the real and imaginary spin-orbit terms share the same $r_{\text {so }}$ and $a_{\text {so }}$ parameters. Therefore the volume-dispersive contribution has different geometry (determined by $r_{v}$ and $a_{v}$ ) from the real smooth volume potential (determined by $r_{\mathrm{HF}}$ and $\left.a_{\mathrm{HF}}\right)$. As a result we have two separate volume contributions to the potential [as can be seen in the first and second line of the Eq. (2)], effectively giving us more flexibility than that allowed by the OMP used in our previous work [22].

It is known that the energy dependence of the depth $V_{\mathrm{HF}}(E)$ is due to the replacement of a microscopic nonlocal HF potential by a local equivalent. For a Gaussian nonlocality $V_{\mathrm{HF}}(E)$ is a linear function of $E$ for large-negative $E$ and is an exponential for large-positive $E$. From Mahaux and Sartor [11], the energy dependence of the HF part of the nuclear mean field is taken as that found by Lipperheide [14]:

$$
V_{\mathrm{HF}}(E)=A_{\mathrm{HF}} \exp \left[-\lambda_{\mathrm{HF}}\left(E-E_{F}\right)\right]
$$

where $A_{\mathrm{HF}}$ and $\lambda_{\mathrm{HF}}$ are undetermined constants with the latter associated with nuclear matter nonlocality range. Equation (4) can be used to describe HF potential in the scattering regime [11]. The present optical potential includes relativistic corrections as discussed by Elton [33] and explained in our recent paper [22].

It is useful to represent the variation of surface $W_{s}(E)$ and volume absorption potential $W_{v}(E)$ depth with energy in functional forms suitable for the DOM analysis. An energy dependence for the imaginary-surface term has been suggested by Delaroche et al. [9] to be:

$$
W_{s}(E)=A_{s} \frac{\left(E-E_{F}\right)^{2}}{\left(E-E_{F}\right)^{2}+\left(B_{s}\right)^{2}} \exp \left(-C_{s}\left|E-E_{F}\right|\right),
$$

where $A_{s}, B_{s}$, and $C_{s}$ are undetermined constants.

The isospin dependence of the potential (the Lane term $[29,30])$ was considered in real-surface $V_{\mathrm{HF}}(E)$ and imaginarysurface $W_{s}(E)$ potentials as follow,

$$
\begin{aligned}
A_{\mathrm{HF}} & =V_{0}\left[1+(-1)^{Z^{\prime}+1} \frac{C_{\text {viso }}}{V_{0}} \frac{N-Z}{A}\right], \\
A_{s} & =W_{0}\left[1+(-1)^{Z^{\prime}+1} \frac{C_{\text {wiso }}}{W_{0}} \frac{N-Z}{A}\right],
\end{aligned}
$$

where $V_{0}, C_{\text {viso }}, W_{0}$ and $C_{\text {wiso }}$ are undetermined constants.

An energy dependence for the imaginary volume term has been suggested in studies of nuclear matter theory by Brown and Rho [34]:

$$
W_{v}(E)=A_{v} \frac{\left(E-E_{F}\right)^{2}}{\left(E-E_{F}\right)^{2}+\left(B_{v}\right)^{2}},
$$

where $A_{v}$ and $B_{v}$ are undetermined constants. The assumption that the imaginary potential $W_{v}(E)$ is symmetric about $E^{\prime}=$ $E_{F}$ is plausible for small values of $\left|E^{\prime}-E_{F}\right|$; however, as was pointed out by Mahaux and Sartor [11], this approximate symmetry no longer holds for large values of $\left|E^{\prime}-E_{F}\right|$. In fact, the influence of the nonlocality of the imaginary part of the microscopic mean field will produce an increase of 
the empirical imaginary part $W\left(r, E^{\prime}\right)$ at large-positive $E^{\prime}$ and approaches zero at large-negative $E^{\prime}[7,35]$. The DOM analysis of neutron scattering on ${ }^{27} \mathrm{Al}$ [36] and ${ }^{232} \mathrm{Th}$ [22] showed the importance of the dispersive contribution to describe $\sigma_{T}$ data for energies above $100 \mathrm{MeV}$ by use of the nonsymmetric version of the volume absorptive potential for large-positive and large-negative energies. Following Mahaux and Sartor [11], we assume that the absorption strengths are modified only outside some fixed energy interval around the Fermi energy $\left[E_{F}-E_{a}, E_{F}+E_{a}\right]$. They used $E_{a}=60 \mathrm{MeV}$; however, this value is fairly arbitrary [11] and we use it as a fitting parameter. Let us assume that the nonlocal imaginary potential to be used in the dispersive integral is denoted by $\widetilde{W}_{v}(E)$; then we can write [12]

$$
\begin{aligned}
\widetilde{W}_{v}(E)= & W_{v}(E)-W_{v}(E) \frac{\left(E_{F}-E-E_{a}\right)^{2}}{\left(E_{F}-E-E_{a}\right)^{2}+E_{a}^{2}}, \\
& \quad \text { for } E<E_{F}-E_{a}, \\
\widetilde{W}_{v}(E)= & W_{v}(E)+\alpha\left[\sqrt{E}+\frac{\left(E_{F}+E_{a}\right)^{3 / 2}}{2 E}\right. \\
& \left.-\frac{3}{2} \sqrt{\left(E_{F}+E_{a}\right)}\right], \quad \text { for } E>E_{F}+E_{a} .
\end{aligned}
$$

These functional forms are chosen in such a way that the function and its first derivative are continuous at $E^{\prime}=\mid E_{F}-$ $E_{a} \mid$. At large-positive energies nucleons sense the "hard-core" repulsive region of the nucleon-nucleon interaction and $\widetilde{W}_{v}(E)$ diverges like $\alpha \sqrt{E}$. Using a model of a dilute Fermi gas hard sphere we can estimate the coefficient $\alpha$ to be equal to $1.65 \mathrm{MeV}^{1 / 2}$ [35], assuming that the Fermi impulse $k_{F}$ is equal to $1.36 \mathrm{fm}^{-1}$ and the radius of the repulsive hard core is equal to $0.4 \mathrm{fm}$. On the contrary, at large-negative energies the volume absorption decreases and goes asymptotically to zero.

In a dispersion relation treatment, the real potential strength consists of a term that varies slowly with energy, the so-called $\mathrm{HF}$ term, $V_{\mathrm{HF}}(\mathbf{r}, E)$, plus a correction term, $\triangle V(\mathbf{r}, E)$, which is calculated with a dispersion relation. Under favorable conditions of analiticity in the complex $E$ plane the real part $\Delta V$ can be constructed from the knowledge of the imaginary part $W$ on the real axis through the dispersion relation

$$
\Delta V(\mathbf{r}, E)=\frac{\mathcal{P}}{\pi} \int_{-\infty}^{\infty} \frac{W\left(\mathbf{r}, E^{\prime}\right)}{E^{\prime}-E} d E^{\prime},
$$

where we have now explicitely indicated the radial and energy dependences of these quantities and $\mathcal{P}$ means that the principal value of the integral should be taken. To simplify the problem, the geometry of the imaginary terms of the OMP are usually assumed to be energy independent and they are expressed in terms of a Woods-Saxon function $f_{\mathrm{ws}}\left[r, R_{i}\left(\theta^{\prime}, \varphi^{\prime}\right)\right]$ or its derivative $g_{\text {ws }}\left[r, R_{i}\left(\theta^{\prime}, \varphi^{\prime}\right)\right]$. In such a case the radial functions factorize out of the integrals and the energy dependence is completely accounted for by two overall multiplicative strenghts $\Delta V(E)$ and $W(E)$. Both of these factors contain, we note, volume and surface contributions. The dispersive treatment employed in this work to calculate dispersive contributions $\Delta V_{v}(E), \Delta V_{s}(E)$, and $\Delta V_{\text {so }}(E)$ was described in detail before [22], so we refer interested readers to that contribution.

\section{SUMMARY OF THE EXPERIMENTAL DATABASES AND PARAMETER FITTING}

A survey of the experimental data for the nucleon interaction on ${ }^{232} \mathrm{Th}$ and ${ }^{238} \mathrm{U}$ nuclei spanning from 0.001 to $200 \mathrm{MeV}$ used in the DCCOM analyses coincides with the data used by Soukhovitskii and co-workers [24]. Additional average total cross-section data for ${ }^{232} \mathrm{Th}$ in the unresolved resonance region from 1 up to $140 \mathrm{keV}$ were recently obtained by Geel's group [37]. The total cross-section data considered cover all the critical energy points that are necessary for revealing the structure that is due to the Ramsauer effect. Energy-averaged total cross sections $\sigma_{T}$ for both nuclei obtained from Abfalterer et al. [6] from 5 to $200 \mathrm{MeV}$ were used to calculate $R_{232} \mathrm{Th}-{ }^{238} \mathrm{U}$ and its associated experimental error.

The potential parameters were searched to reproduce available neutron- and proton-induced cross section data for ${ }^{232} \mathrm{Th}$ and ${ }^{238} \mathrm{U}$. At incident energies above several mega-electronvolts, it is almost impossible to separate neutron-inelasticscattering data into each excitation level experimentally for actinide nucleus. Thus experimental proton-scattering data, which can be done with much higher resolution, were highly relevant for determining the optical potential parameters uniquely. Evaluated neutron strength functions for ${ }^{232} \mathrm{Th}$ and ${ }^{238} \mathrm{U}, S_{0}$ and $S_{1}$, and potential scattering radius $R^{\prime}[37,38]$ were used in parameter search.

We searched for the optical potential parameters by minimizing the quantity $\chi^{2}$ defined by

$$
\begin{aligned}
\chi^{2}= & \frac{1}{N+M+3}\left[\sum_{i=0,1}\left(\frac{S_{i, \text { calc }}-S_{i, \text { eval }}}{\Delta S_{i, \text { eval }}}\right)^{2}+\left(\frac{R_{\text {calc }}^{\prime}-R_{\text {eval }}^{\prime}}{\Delta R_{\text {eval }}^{\prime}}\right)^{2}\right. \\
& +\sum_{i=1}^{N} \frac{1}{K_{i}} \sum_{j=1}^{K_{i}}\left(\frac{d \sigma_{i j} / d \Omega_{\text {calc }}-d \sigma_{i j} / d \Omega_{\text {exp }}}{\Delta d \sigma_{i j} / d \Omega_{\text {exp }}}\right)^{2} \\
& +\sum_{i=1}^{M}\left(\frac{\left.\left.\sigma_{\text {tot }_{\text {calc }_{i}}}-\sigma_{\text {tot }_{\text {eval }_{i}}}\right)^{2}\right]}{\Delta \sigma_{\text {tot }_{\text {eval }_{i}}}}\right)^{2}
\end{aligned}
$$

where $N$ denotes number of experimental scattering data sets, $K_{i}$ is the number of angular points in each scattering data set, and $M$ is the number of energies for which the experimental (evaluated) neutron total cross section is involved.

The optical model code OPTMAN $[39,40]$ was used for OMP parameter fitting. Originally the code did not include dispersion terms, so numerical [41] and analytical solutions $[42,43]$ of dispersion relations were implemented within the OPTMAN code. We used symmetric surface and nonsymmetric volume imaginary absorptive potentials; therefore we initially adjusted 16 parameters, namely $V_{0}, \lambda_{\mathrm{HF}}$, and $C_{\mathrm{viso}}$, which define the smooth energy dependence of the real volume potential; $W_{0}, C_{\text {wiso }}, B_{s}, C_{s}$ and $A_{v}, B_{v}, E_{a}$, which define the surface and volume absorptive potentials, respectively; and six geometrical parameters $\left(r_{\mathrm{HF}}, a_{\mathrm{HF}}, r_{v}, a_{v}, r_{s}, a_{s}\right)$. After proper values were obtained by these global minimization spin-orbit parameters $V_{\text {so }}, \lambda_{\text {so }}, A_{\text {so }}, B_{\text {so }}, r_{\text {so }}$, and $a_{\text {so }}$, parameters of the Coulomb interaction, $C_{\mathrm{Coul}}, r_{c}$, and $a_{c}$ and multipolar deformation parameters $\beta_{i}$ (for both nuclei) were also optimized. The final iteration involved a free variation of all parameters by use of the best-fit search option of the OPTMAN code. 
TABLE I. Dispersive coupled-channel OMP parameters for ${ }^{238} \mathrm{U}\left({ }^{232} \mathrm{Th}\right)$. We used the following deformation parameters: $\beta_{2}=$ $0.228, \beta_{4}=0.062, \beta_{6}=-0.0056$ for ${ }^{238} \mathrm{U}$ and $\beta_{2}=0.213, \beta_{4}=0.069, \beta_{6}=0.0017$ for ${ }^{232} \mathrm{Th}$.

\begin{tabular}{|c|c|c|c|c|}
\hline & VOLUME & SURFACE & SPIN-ORBIT & COULOMB \\
\hline $\begin{array}{c}\text { Real depth } \\
(\mathrm{MeV})\end{array}$ & $\begin{aligned} V_{0} & =48.62 \\
\lambda_{\mathrm{HF}} & =0.01037 \\
C_{\text {viso }} & =10.0\end{aligned}$ & Dispersive & $\begin{array}{l}V_{\mathrm{so}}=6.03 \\
\lambda_{\mathrm{so}}=0.005\end{array}$ & $C_{\text {Coul }}=1.62$ \\
\hline $\begin{array}{c}\text { Imaginary depth } \\
(\mathrm{MeV})\end{array}$ & $\begin{array}{l}A_{v}=12.53 \\
B_{v}=80.94 \\
E_{a}=350\end{array}$ & $\begin{aligned} W_{0} & =17.73 \\
B_{s} & =11.56 \\
C_{s} & =0.01328 \\
C_{\text {wiso }} & =23.5\end{aligned}$ & $\begin{array}{c}W_{\mathrm{so}}=-3.1 \\
B_{\mathrm{so}}=160\end{array}$ & \\
\hline $\begin{array}{l}\text { Geometry } \\
\quad(\mathrm{fm})\end{array}$ & $\begin{aligned} r_{\mathrm{HF}} & =1.2516(1.2598) \\
a_{\mathrm{HF}} & =0.636(0.624) \\
r_{v} & =1.253 \\
a_{v} & =0.680(0.678)\end{aligned}$ & $\begin{array}{l}r_{s}=1.1808 \\
a_{s}=0.603(0.600)\end{array}$ & $\begin{array}{l}r_{\mathrm{so}}=1.1214 \\
a_{\mathrm{so}}=0.59\end{array}$ & $\begin{array}{l}r_{c}=1.2174 \\
a_{c}=0.551\end{array}$ \\
\hline
\end{tabular}

All parameters but deformation and geometrical ones were exactly the same for both ${ }^{232} \mathrm{Th}$ and ${ }^{238} \mathrm{U}$ nuclei fitting. The derived DCCOM potential parameters are listed in Table I. The attained minimum $\chi^{2}$ value, as defined by Eq. (12) for all the available ${ }^{238} \mathrm{U}\left({ }^{232} \mathrm{Th}\right)$ experimental data is 2.10 (2.55), with 2.00 (2.21) for the neutron database, and for the proton angular data description the corresponding $\chi^{2}$ minimum is 2.39 (3.52). These values compared favorably with the NDOMP04 values, which are 1.66 (2.55) and 1.90 (3.8) for neutron and proton databases, respectively. One can see that the $\chi^{2}$ value is slightly increased for ${ }^{238} \mathrm{U}$ and decreased for ${ }^{232} \mathrm{Th}$, probably because a new accurate Geel's group data [37] was used in the optical parameter search for the latter.

\section{RESULTS AND DISCUSSION}

Optical model calculations of the total cross section for ${ }^{232} \mathrm{Th}$ and ${ }^{238} \mathrm{U}$ nuclei were carried out with the newly fitted DCCOM potential, NDOMP04, and the OMPs of Ignatyuk et al. [26,28], Maslov [5], and Maslov et al. [25]. The calculated total neutron cross-section data were used to obtain the ratio $R_{232}{ }^{2 h-}{ }^{238} \mathrm{U}$ for each of employed potentials. Calculated results were compared with the experimental data of Abfalterer et al. [6] in Fig. 1. The experimental data was shifted up by +0.002 , well within the estimated uncertainty of the vertical scale (0.02) [4], arising from uncertainties in the areal densities of the uranium and thorium targets. The measurements are well reproduced by the axial rigid-rotor DCCOM potential calculations with the present optical potential parameters from Table I. Conventional nondispersive $\mathrm{CC}$ potentials fail to reproduce the experimental data; the NDOMP04 results are closer to the data than results for the other two potentials.

The CC model also makes it possible to calculate the angular distributions for scattering of neutrons and protons to the low-lying collective levels belonging to the ground-state rotational band of ${ }^{232} \mathrm{Th}$ and ${ }^{238} \mathrm{U}$ nuclei. We are not showing pictures, but the quality of the agreement is excellent as can be judged by the obtained $\chi^{2}$. Furthermore, calculated average resonance parameters reproduce the evaluated values for ${ }^{232} \mathrm{Th}$ and ${ }^{238} \mathrm{U}$ nuclei very well, as shown in Table II. It is important to remark on the advantage of the dispersive approach for average resonance parameters' description. The real potential of this work is almost flat toward the low energies, by a combination of the increasing contribution of the smooth exponential $\mathrm{HF} V_{\mathrm{HF}}(E)$ potential with the decreasing dispersive contribution (which goes to zero at Fermi energy). Such behavior allows for a good simultaneous description of the average low-energy total cross sections, strength functions, and scattering radius. This simultaneous description is a tough challenge for conventional nondispersive potentials.

The derived optical potential energy dependence is very simple. We do not need to introduce energy-dependent geometry, and the potential parameters are unique for both neutron and proton projectiles. The dispersion relations, coupled to the smooth energy-dependent $\mathrm{HF}$ potential $V_{\mathrm{HF}}(E)$, fully determine the real part of the dispersive contribution once the imaginary part of the mean field is fixed. Very few parameters are required in comparison with a conventional nondispersive CC OMP analysis.

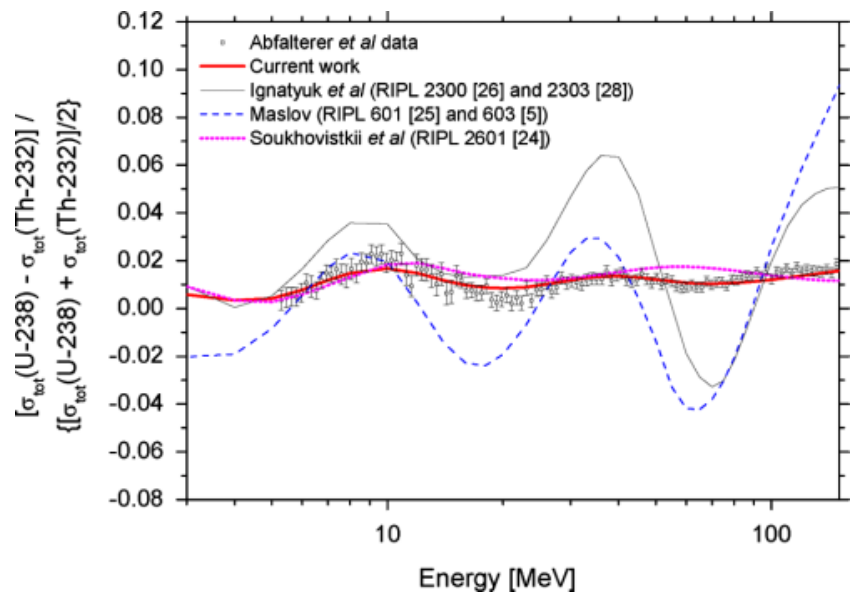

FIG. 1. (Color online) Energy dependence of the measured ratio $\left[\sigma\left({ }^{238} \mathrm{U}\right)-\sigma\left({ }^{232} \mathrm{Th}\right)\right] /\left\{\left[\sigma\left({ }^{232} \mathrm{Th}\right)+\sigma\left({ }^{238} \mathrm{U}\right)\right] / 2\right\}$ versus calculated values obtained with different OMPs (the corresponding RIPL OMP index [38] is indicated in parentheses). 
TABLE II. Comparison of predicted resonance parameters with experimental ones.

\begin{tabular}{|c|c|c|c|c|c|c|}
\hline \multirow[b]{2}{*}{ Nuclei } & \multicolumn{3}{|c|}{ Present work (evaluated at $2 \mathrm{keV}$ ) } & \multicolumn{3}{|c|}{ Evaluated values } \\
\hline & $S_{0},(\mathrm{eV})^{-1 / 2} 10^{-4}$ & $S_{1},(\mathrm{eV})^{-1 / 2} 10^{-4}$ & $R^{\prime}(\mathrm{fm})$ & $S_{0},(\mathrm{eV})^{-1 / 2} 10^{-4}$ & $S_{1},(\mathrm{eV})^{-1 / 2} 10^{-4}$ & $R^{\prime}(\mathrm{fm})$ \\
\hline${ }^{238} \mathrm{U}$ & 0.918 & 1.72 & 9.59 & $1.03(0.08)[38]$ & $1.6(0.4)[38]$ & $9.6(0.1)[24]$ \\
\hline${ }^{232} \mathrm{Th}$ & 0.859 & 1.72 & 9.62 & $\begin{array}{l}0.935(0.05)[37] \\
0.87(0.07)[38]\end{array}$ & $1.81(0.03)[37]$ & $9.53(0.05)$ [37] \\
\hline
\end{tabular}

\section{CONCLUSIONS}

In this work we have presented a dispersive isospindependent relativistic $\mathrm{CC}$ optical model analysis of nucleon scattering on ${ }^{232} \mathrm{Th}$ and ${ }^{238} \mathrm{U}$ nuclei from $1 \mathrm{keV}$ to $200 \mathrm{MeV}$. The use of proton- and neutron-scattering data simultaneously made it possible to increase the accuracy of estimated optical potential parameters, especially in the high-energy region. The excellent overall agreement obtained between predictions and experimental data would not have been possible without including dispersive terms in the calculations and nonlocality effects in the volume absorptive potential. Very weak dependence of the geometrical parameters on mass number is observed. Calculations that use the DCCOM potential reproduce the experimental total cross-section differ- ence between ${ }^{232} \mathrm{Th}$ and ${ }^{238} \mathrm{U}$ nuclei measured by Abfalterer et al. [6] within experimental uncertainty. The isovector terms and the very weak dependence of the geometrical parameters on mass number $A$ give the possibility of extending the derived potential parameters to neighboring actinide nuclei with a great confidence. This work is in progress.

\section{ACKNOWLEDGMENTS}

R.C. is grateful to A. Trkov for encouraging discussions and comments. This work was partially supported by International Atomic Energy Agency, through IAEA Research Contract 13067 and by Japan under ISTC Project B-521.
[1] R. E. Shamu, E. M. Bernstein, J. J. Ramirez, and Ch. Lagrange, Phys. Rev. C 22, 1857 (1980).

[2] H. S. Camarda, T. W. Phillips, and R. M. White, Phys. Rev. C 29, 2106 (1984).

[3] H. S. Camarda, F. S. Dietrich, and T. W. Phillips, Phys. Rev. C 39, 1725 (1986).

[4] F. S. Dietrich, J. D Anderson, R. W. Bauer, S. M. Grimes, R. W. Finlay, W. P. Abfalterer, F. B. Bateman, R. C. Haight, G. L. Morgan, E. Bauge, J. P. Delaroche, and P. Romain, Phys. Rev. C 67, 044606 (2003).

[5] V. M. Maslov, Nucl. Phys. A757, 390 (2005).

[6] W. P. Abfalterer, F. B. Bateman, F. S. Dietrich, R. W. Finlay, R. C. Haight, and G. L. Morgan, Phys. Rev. C 63, 044608 (2001).

[7] C. Mahaux and H. Ngô, Nucl. Phys. A431, 486 (1984).

[8] C. Mahaux, H. Ngô, and G. R. Satchler, Nucl. Phys. A449, 354 (1986).

[9] J. P. Delaroche, Y. Wang, and J. Rapaport, Phys. Rev. C 39, 391 (1989).

[10] W. Tornow, Z. P. Chen, and J. P. Delaroche, Phys. Rev. C 42, 693 (1990).

[11] C. Mahaux and R. Sartor, Nucl. Phys. A528, 253 (1991).

[12] C. Mahaux and R. Sartor, Advances in Nuclear Physics, edited by J. W. Negele and E. Vogt (Plenum, New York, 1991), Vol. 20.

[13] R. Lipperheide, Nucl. Phys. A89, 97 (1966).

[14] R. Lipperheide, Z. Phys. 202, 58 (1967).

[15] G. Passatore, Nucl. Phys. A95, 694 (1967).

[16] R. Lipperheide and A. K. Schmidt, Nucl. Phys. A112, 65 (1968).

[17] B. Morillon and P. Romain, Phys. Rev. C 70, 014601 (2004).

[18] P. Romain and J. P. Delaroche, A dispersive coupled channel analysis of nucleon scattering from ${ }^{181} \mathrm{Ta}$ and ${ }^{182,184,186} \mathrm{~W}$ up to $200 \mathrm{MeV}$. In Proceedings of the Meeting on Nucleon-Nucleus Optical Model up to $200 \mathrm{MeV}$, Bruyères-le-Châtel,p. 167. Avail- able on-line at http://www.nea.fr/html/science/om200/, OECD, Paris, 1997.

[19] A. B. Smith, Ann. Nucl. Energy 28, 1745 (2001).

[20] A. B. Smith, Ann. Nucl. Energy 29, 1241 (2002).

[21] A. B. Smith, Ann. Nucl. Energy 31, 1813 (2004).

[22] E.Sh. Soukhovitskiî, R. Capote, J. M. Quesada, and S. Chiba, Phys. Rev. C 72, 024604 (2005).

[23] Ch. Lagrange, in Critique of Nuclear Models and Their Validity in the Evaluation of Nuclear Data, p. 58. Technical Report JAERI-M-5984, NEANDC(J)-38, INDUC(JAP)-25, Japan Atomic Energy Research Institute, 1975.

[24] E. Sh. Soukhovitskĩi, S. Chiba, J-Y. Lee, O. Iwamoto, and T. Fukahori, J. Phys. G 30, 905 (2004).

[25] V. M. Maslov, Y. V. Porodzinskiĩ, N. A. Tetereva, M. Baba, and A. Hasegawa, Nucl. Phys. A736, 77 (2004).

[26] A. V. Ignatyuk, V. P. Lunev, Yu. N. Shubin, E. V. Gai, N. N. Titarenko, A. Ventura, and W. Gudowski, Nucl. Sci. Eng. 136, 340 (2000).

[27] A. Trkov, Technical Report INDC(NDS)-447, IAEA, Vienna, 2003. Available online at http://www-nds.iaea.org/reports/indcnds-447.pdf.

[28] A. V. Ignatyuk, E. V. Gai, V. P. Lunev, Yu. N. Shubin, and N. N. Titarenko, Private Communication. As quoted in Technical Report IAEA RC 12571, IAEA, Vienna, 2004.

[29] A. M. Lane, Phys. Rev. Lett. 8, 171 (1962).

[30] A. M. Lane, Nucl. Phys. 35, 676 (1962).

[31] R. H. Bassel, R. M. Drisko, and G. R. Satchler, The distortedwave theory of direct nuclear reactions. Technical Report ORNL-3240, Oak Ridge National Laboratory, Oak Ridge, TN, 1962.

[32] S. Chiba, O. Iwamoto, Y. Yamanouti, M. Sugimoto, M. Mizumoto, K. Hasegawa, E. S. Sukhovitskiĩ, Y. V. Porodzinskiĩ, and Y. Watanabe, Nucl. Phys. A624, 305 (1997). 
[33] L. R. B. Elton, Nuovo Cimento B 42, 227 (1966).

[34] G. E. Brown and M. Rho, Nucl. Phys. A372, 397 (1981).

[35] C. Mahaux and R. Sartor, Nucl. Phys. A458, 25 (1986).

[36] A. Molina, R. Capote, J. M. Quesada, and M. Lozano, Phys. Rev. C 65, 034616 (2002).

[37] K. Volev, N. Koyumdjieva, A. Brusegan, A. Borella, P. Siegler, N. Janeva, A. Lukyanov, L. Leal, and P. Schillebeeckx, Evaluation of the ${ }^{232} \mathrm{Th}$ neutron cross sections between $4 \mathrm{keV}$ and $140 \mathrm{keV}$. In Proceedings of the International Conference on Nuclear Data for Science and Technology, 27 September1 October 2004, Santa Fé, NM, USA; also published in AIP Conf. Proc. 769, 87 (2005).

[38] T. Belgya, O. Bersillon, R. Capote, T. Fukahori, G. Zhigang, S. Goriely, M. Herman, A. V. Ignatyuk, S. Kailas, A. Koning, P. Oblozhinsky, V. Plujko, and P. Young, Handbook For Calculations of Nuclear Reaction Data: Reference Input Parameter Library. Available on-line at http://www-nds.iaea.org/RIPL-2/, IAEA, Vienna, 2005.
[39] E. Sh. Soukhovitskiĩ, S. Chiba, O. Iwamoto, K. Shibata, T. Fukahori, and G. B. Morogovskiñ, Programs OPTMAN and SHEMMAN Version 8. Technical Report JAERI-Data/Code 2005-002 (Japan Atomic Energy Research Institute, Ibaraki, Japapn, 2005).

[40] E. Sh. Soukhovitskiĩ, G. B. Morogovskiĩ, S. Chiba, O. Iwamoto, and T. Fukahori, Physics and Numerical Methods of OPTMAN: A Coupled-channels Method Based on Soft-rotator Model for a Description of Collective Nuclear Structure and Excitation. Technical Report JAERI-Data/Code 2004002 (Japan Atomic Energy Research Institute, Ibaraki, Japan, 2004).

[41] R. Capote, A. Molina, and J. M. Quesada, J. Phys. G 27, B 15 (2001).

[42] J. M. Quesada, R. Capote, A. Molina, M. Lozano, and J. Raynal, Phys. Rev. C 67, 067601 (2003).

[43] J. M. Quesada, R. Capote, A. Molina, and M. Lozano, Comput. Phys. Commun. 153, 97 (2003). 